\title{
Macrofauna edáfica associada a plantas de cobertura em plantio direto em um Latossolo Vermelho do Cerrado
}

\author{
Glenio Guimarães Santos ${ }^{(1)}$, Pedro Marques da Silveira ${ }^{(2)}$, Robélio Leandro Marchão ${ }^{(3)}$, Thierry Becquer ${ }^{(4)}$ \\ e Luiz Carlos Balbino(5)
}

\begin{abstract}
(1)Universidade Federal de Goiás, Escola de Agronomia e Engenharia de Alimentos, Caixa Postal 131, CEP 74001-970 Goiânia, GO. E-mail: gleniogm@gmail.com (2)Embrapa Arroz e Feijão, Caixa Postal 179, CEP 75375-000 Santo Antônio de Goiás, GO. E-mail: pmarques@cnpaf.embrapa.br (3)Embrapa Cerrados, Caixa Postal 08223, CEP 73310-970 Planaltina, DF. E-mail: robelio.leandro@cpac.embrapa.br (4)Institut de Recherche pour le Développement, Unité Mixte de Recherche 137, SupAgro, 2 Place Viala, Bâtiment 12, 34060 Montpellier Cedex 2, França. E-mail: becquer@cpac.embrapa.br (5)Embrapa Transferência de Tecnologia, Parque Estação Biológica, Embrapa Sede, CEP 70770-901 Brasília, DF. E-mail: luizcarlos.balbino@embrapa.br
\end{abstract}

\begin{abstract}
Resumo - O objetivo deste trabalho foi caracterizar a macrofauna edáfica e avaliar o efeito de plantas de cobertura em plantio direto, nos principais grupos da macrofauna do solo, em duas épocas de avaliação em um Latossolo Vermelho distroférrico. O delineamento experimental foi o de blocos ao acaso, com oito tratamentos (plantas de cobertura) e quatro repetições. As plantas de cobertura: Crotalaria juncea, guandu-anão (Cajanus cajan), Stylosanthes guianensis, Brachiaria brizantha, B. brizantha consorciada com milho (Zea mays), milheto (Pennisetum glaucum), mombaça (Panicum maximum) e Sorghum bicolor foram cultivadas de novembro a abril. Em setembro de cada ano, foi realizado o plantio de feijão, em cultivo irrigado por pivô central. A área útil em cada parcela foi de $60 \mathrm{~m}^{2}$. Amostras de solo na forma de monólitos $(25 \times 25 \mathrm{~cm})$ foram retiradas aleatoriamente em cada parcela, para contagem da macrofauna, às profundidades de $0-10 \mathrm{~cm}$ e $10-20 \mathrm{~cm}$, em abril e em setembro de 2005. Os grupos taxonômicos, identificados em ordem decrescente de densidade relativa, são: Formicidae, Oligochaeta, Dermaptera, Coleoptera, Hemiptera, Miriapoda, Isoptera, Araneae, Lepidoptera, Blattodea e larvas de Diptera. Crotalaria juncea apresentou maior densidade de macrofauna, seguida por B. Brizantha, B. Brizantha consorciada com milho, Sorghum bicolor, Stylosanthes guianensis, Cajanus Cajans, Pennisetum Glaucum e Panicum maximum. O uso das plantas de cobertura, associado à irrigação na avaliação de setembro, favorece a colonização do solo pela macrofauna.
\end{abstract}

Termos para indexação: biologia do solo, gramíneas tropicais, invertebrados do solo, qualidade do solo, rotação de culturas.

\section{Soil macrofauna communities and cover crops in a Cerrado Oxisol under no tillage}

\begin{abstract}
The objective of this work was to characterize soil fauna groups and to evaluate the effects of cover crops under no-tillage system, in a Cerrado Oxisol, in two evaluation periods. The cover crops: Crotalaria juncea, Cajanus cajan, Stylosanthes guianensis, Brachiaria brizantha, Brachiaria brizanthalZea mays association, Pennisetum glaucum, Panicum maximum and Sorghum bicolor were cultivated from November to April of each year. Common bean was sown every September under centre pivot irrigation. The area of each plot was $60 \mathrm{~m}^{2}$. Soil monoliths $(25 \times 25 \mathrm{~cm})$ were taken randomly from each plot, at 0-10 cm and 10-20 cm dephs, in April and September, 2005, and macrofauna was hand sorted. Identified groups in decreasing order of total density are: Formicidae, Oligochaeta, Dermaptera, Coleoptera, Hemiptera, Miriapoda, Isoptera, Araneae, Lepidoptera, Blattodea and Diptera larvae. Crotalaria juncea showed the highest macrofauna density, followed by B. brizantha, B. brizantha/Zea mays association, Sorghum bicolor, Stylosanthes guianensis, Cajanus cajans, Pennisetum. glaucum, Panicum maximum. The use of cover species in no-tillage system, associated with irrigation, promotes soil colonization by macrofauna.
\end{abstract}

Index terms: soil biology, tropical gramineous, soil invertebrates, soil quality, crop rotation.

\section{Introdução}

A utilização de plantas de cobertura, na forma de adubo verde, é uma das alternativas para o manejo sustentável dos solos (Alvarenga et al., 1995), especialmente do Cerrado (Amábile et al., 2000). A adubação verde associada à rotação de culturas, com incorporação de plantas de cobertura ao solo, favorece a reciclagem de nutrientes, a agregação, o armazenamento da água e a manutenção da matéria orgânica do solo, em comparação com os monocultivos anuais, com efeitos positivos na fertilidade e reciclagem de nutrientes (Alcântara et al., 2000; Boer et al., 2007). 
O sistema plantio direto (SPD) e os sistemas de integração lavoura-pecuária (SILP) são também alternativas de manejo que conciliam a manutenção e até mesmo a elevação da produtividade, com maior racionalidade dos insumos empregados. O sucesso desses sistemas no Cerrado deve-se ao fato de que a palhada, acumulada pelas plantas de cobertura ou das pastagens, e restos culturais de lavouras comerciais proporcionam um ambiente favorável à recuperação ou à manutenção das propriedades biológicas do solo (Menezes et al., 2004) e favorecem, também, as comunidades da macrofauna edáfica (Marchão, 2007; Silva et al., 2007).

A presença de cobertura morta no SPD e no SILP estimula a fauna edáfica, as raízes e a microflora do solo, o que permite manter o solo em equilíbrio e permanentemente protegido contra a degradação (Lavelle \& Spain, 2001). Da mesma forma, a manutenção de uma cobertura vegetal na superfície do solo impede a perda da diversidade da macrofauna edáfica e favorece a atividade dos organismos engenheiros do ecossistema, entre eles os grupos Oligochaeta, Formicidae e Isoptera (Barros et al., 2003). A macrofauna tem diferentes efeitos nos processos que condicionam a fertilidade do solo, pela regulação das populações microbianas responsáveis pela humificação e mineralização (Lavelle et al., 1997) e pela formação de agregados, que podem proteger parte da matéria orgânica do solo de uma mineralização rápida, por meio de sua ação mecânica, como os Oligochaeta, Formicidae e Isoptera (Lee, 1994).

Apesar de ser conhecida a importância da macrofauna edáfica para o equilíbrio e funcionamento dos ecossistemas, poucos estudos têm sido realizados, especialmente no bioma Cerrado, para se avaliar efeitos das práticas de manejo sobre esses organismos. Pouco se sabe quanto ao papel das plantas de cobertura utilizadas como adubo verde ou associadas ao SPD, sobre a dinâmica das comunidades de invertebrados do solo. Muitos estudos têm destacado os benefícios da utilização da adubação verde, especialmente com espécies leguminosas, na sustentabilidade e manutenção das propriedades do solo, contudo, os mecanismos responsáveis por estes benefícios ainda não são completamente conhecidos. Grande parte dos trabalhos realizados sobre a biologia dos solos do Cerrado foram direcionados à microflora e microfauna e, segundo Blanchart et al. (2006), em regiões tropicais, a caracterização da atividade biológica e diversidade do solo pode ajudar a compreender a dinâmica da estrutura do solo e o fluxo de nutrientes no sistema solo-planta.

Este trabalho teve como objetivo caracterizar a macrofauna edáfica e avaliar o efeito de plantas de cobertura em plantio direto, nos principais grupos da macrofauna do solo, em diferentes épocas de avaliação, em um Latossolo Vermelho distroférrico.

\section{Material e Métodos}

O experimento foi instalado na área experimental da Fazenda Capivara, Embrapa Arroz e Feijão, no Município de Santo Antônio de Goiás, GO, em um Latossolo Vermelho distroférrico (Embrapa, 2006), textura argilosa, fase Cerradão subperenifólio e relevo plano.

A vegetação nativa da área em estudo foi removida em 1983, com instalação de pivô central no mesmo ano e, desde o primeiro cultivo, foi manejada com diversas rotações entre plantas gramíneas e leguminosas, de interesse comercial e experimental. Para formar cobertura para o cultivo do feijão (maio-setembro) sob pivô central, desde 2001 - com a implantação do experimento -, têm sido cultivadas em SPD (setembro-março) as seguintes plantas: braquiária solteira (Brachiaria brizantha cv. Marandu); braquiária consorciada com milho em SILP (Zea mays L. cv. BRS $3150+$ B. brizantha); guanduanão (Cajanus cajan L. Millisp); milheto (Pennisetum glaucum (L.) R. Br. cv. BN-2); capim-mombaça (Panicum maximum cv. Mombaça); sorgo granífero (Sorghum bicolor L. Moench cv. BR 304); e estilosantes (Stylosanthes guianensis cv. Mineirão). O delineamento experimental utilizado foi o de blocos ao acaso, com oito tratamentos e quatro repetições. O tamanho de cada parcela foi de $6 \mathrm{~m}$ de largura por $10 \mathrm{~m}$ de comprimento.

As plantas de cobertura foram semeadas em novembro de 2004. A adubação de plantio utilizada, para todas as culturas de cobertura, foi de $400 \mathrm{~kg} \mathrm{ha}^{-1}$ da fórmula 4-30-16. Em dezembro de 2004, foram aplicados $30 \mathrm{~kg} \mathrm{ha}^{-1}$ de nitrogênio na forma de sulfato de amônio, nas parcelas de braquiária consorciada com milho. Em abril de 2005, foi realizado o corte das plantas de cobertura, com um triturador de palha, e o material vegetal foi distribuído uniformemente sobre o solo. Em maio de 2005 , foram aplicados $4 \mathrm{~L} \mathrm{ha}^{-1}$ de glyphosate, em toda área do experimento, para dessecação da área. Em junho de 2005, foi realizado o plantio de feijão, cultivar BRS Valente. A adubação de plantio foi de $400 \mathrm{~kg} \mathrm{ha}^{-1} \mathrm{da}$ fórmula 4-30-16 + Zn. 
As amostragens da macrofauna edáfica foram realizadas em duas épocas, em abril e em setembro de 2005, pelo método Tropical Soil Biology and Fertility (TSBF) (Anderson \& Ingram, 1993) modificado. Os monólitos de $25 \times 25 \mathrm{~cm}$ foram retirados na camada de $0-10 \mathrm{~cm}$, inclusive a liteira ou serrapilheira de superfície, e na camada de $10-20 \mathrm{~cm}$, por meio de um quadro amostrador metálico que foi lançado aleatoriamente na parcela. As amostragens de abril e setembro foram realizadas logo após o corte das plantas de cobertura e imediatamente após a colheita do feijão.

As amostras de solo foram acondicionadas em sacos de plástico, foram identificadas e conduzidas ao laboratório para triagem dos macroinvertebrados edáficos, por meio de catação manual. Após a triagem, os invertebrados foram acondicionados em tubos de ensaio de $25 \mathrm{~mL}$, com álcool a $70 \%$, para posterior identificação.

Os invertebrados do solo foram identificados com auxílio de lupa binocular e separados por grandes grupos taxonômicos. Após a contagem, foram determinadas a densidade relativa (número de indivíduos por metro quadrado) e a riqueza de grupos taxonômicos, presentes em cada tratamento. Os dados de densidade relativa (DR) por grupo e riqueza total de grupos taxonômicos, em função das plantas de cobertura, profundidades do solo e épocas de amostragem, foram submetidos ao teste de Kolmogorov-Smirnov. Em razão da ausência de normalidade, e as médias foram transformadas em $(\mathrm{x}+1)^{0,5}$. Para comparação das médias transformadas em função das plantas de cobertura em cada época, utilizou-se o teste de Tukey, a 10\% de probabilidade.

Com auxílio do programa ADE-4 (Thioulouse et al., 1997), realizou-se também uma análise de componentes principais (ACP), para identificar, entre as variáveis correspondentes aos grupos da macrofauna, quais contribuíram com maior peso na combinação linear das duas primeiras componentes principais. Para auxiliar na interpretação dos resultados, além do círculo de correlações entre os autovetores das variáveis, foram construídos diagramas de ordenação dos tratamentos (plantas de cobertura), do efeito do agrupamento em função da família (gramíneas ou leguminosas) e da época de amostragem (abril ou setembro). A análise discriminante, baseada na dissimilaridade ou distância de Mahalanobis, foi utilizada para comparar as distâncias matemáticas entre as amostras no diagrama de ordenação. Este tipo de análise utiliza um teste de permutação, que calcula a inércia total interclasse, para cada distribuição aleatória de indivíduos e, por associação a uma probabilidade estatística, permite aumentar o poder discriminante da análise (Thiolouse et al., 1997).

\section{Resultados e Discussão}

Os grupos taxonômicos, identificados nos tratamentos em ordem decrescente de densidade relativa, foram: Formicidae, Oligochaeta, Dermaptera, Coleoptera (adulto e larva), Hemiptera, Miriapoda, Isoptera, Araneae, Lepidoptera, Blattodea e larvas de Diptera (Tabela 1). A densidade relativa máxima foi observada no tratamento crotalária (1.372 indivíduos $\mathrm{m}^{-2}$ ), enquanto a densidade relativa mínima foi observada no tratamento mombaça (532 indivíduos $\mathrm{m}^{-2}$ ).

Os indivíduos dos grupos Formicidae e Oligochaeta representaram $59,1 \%$ da densidade total da macrofauna do solo, e o grupo Formicidae foi o que apresentou maior DR no solo (3.052 indivíduos, o que representa 36,3\% da fauna total identificada), seguido pelo grupo Oligochaeta com 1.924 indivíduos (22,9\% da fauna total). Silva et al. (2007) observaram resultados semelhantes, com predominância do grupo Formicidae, em áreas de mucuna e milheto no sistema plantio direto, em que este grupo representou $57,3 \%$ da densidade total.

A densidade observada do grupo Isoptera correspondeu a $2,7 \%$ da densidade total da macrofauna. Este resultado difere da maioria dos outros estudos conduzidos no bioma Cerrado, onde foi demonstrado que este grupo é predominante (Dias et al., 1997; Benito et al., 2004; Constantino, 2005; Silva et al., 2007). Normalmente, altas densidades do grupo Isoptera (cupins) são comuns em áreas de pastagens, geralmente com baixa fertilidade química (percentagem de matéria orgânica e alta relação $\mathrm{C} / \mathrm{N}$ ) e ausência de adubação, características que não correspondem ao experimento deste estudo.

A análise de componentes principais (ACP), realizada com os dados de densidade dos principais grupos da macrofauna edáfica, revelou que os dois primeiros eixos explicaram $33,8 \%$ da variabilidade total dos dados, que foram 18,9 e $14,9 \%$ pelo primeiro e segundo eixos, respectivamente (Figura $1 \mathrm{~A}$ ). O eixo 1 foi influenciado, principalmente, pelos grupos Coleoptera (adulto), Oligochaeta, Miriapoda e Hemiptera, que apresentaram autovetores positivos. $\mathrm{O}$ eixo 2 foi influenciado pelos grupos Coleoptera (larva), Isoptera, Diptera, e de uma forma mais limitada, pelo grupo Formicidae, com autovetores positivos. Blattodea e Lepidoptera também 


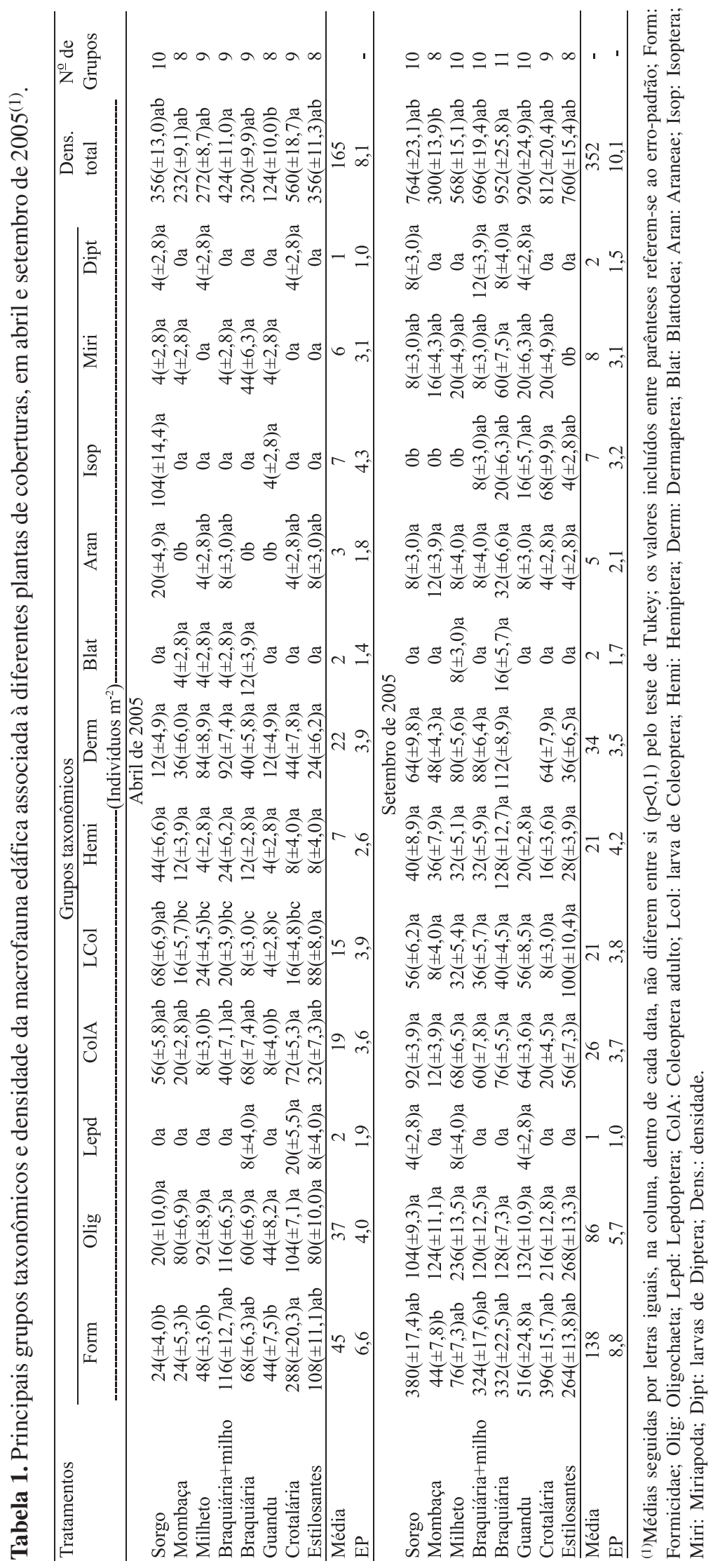


contribuíram com o eixo 2 , porém, com autovetores negativos.

Em relação à presença de grupos, observou-se maior riqueza no tratamento braquiária (10 grupos), enquanto a menor riqueza foi observada no tratamento mombaça (seis grupos). Dias et al. (2006, 2007) ao estudar leguminosas perenes em sistema silvipastoril, observaram que a presença das leguminosas contribuiu para o aumento da diversidade da fauna de solo, o que demonstra que a diversidade vegetal favorece a macrofauna do solo.

No diagrama de ordenação, observa-se o agrupamento dos pontos em função das espécies de plantas de cobertura (Figura 1 B). A separação entre as espécies foi significativa $(p=0,006)$, e observou-se que houve um agrupamento das gramíneas e das leguminosas em relação aos dois primeiros eixos.
Pela ordenação dos pontos, em relação ao eixo 1 , demonstrou-se que os tratamentos sorgo, braquiária e SILP foram os que apresentaram maior diversidade, sobretudo dos grupos de indivíduos habitantes da liteira ou serrapilheira de superfície. Alguns desses grupos, entre eles Araneae, Miriapoda, larvas de Diptera e Coleoptera, são predadores ou responsáveis pela fragmentação da matéria orgânica e apresentam-se como indicadores sensíveis da qualidade do solo (Lavelle \& Spain, 2001). Silva et al. (2006) e Marchão (2007) também observaram que sistemas de integração lavoura-pecuária favoreceram a colonização do solo por alguns grupos da macrofauna, entre eles Coleoptera e Oligochaeta. Os tratamentos mombaça e milheto apresentaram menor abundância de indivíduos. Na ordenação em relação ao eixo 2, observa-se que os grupos

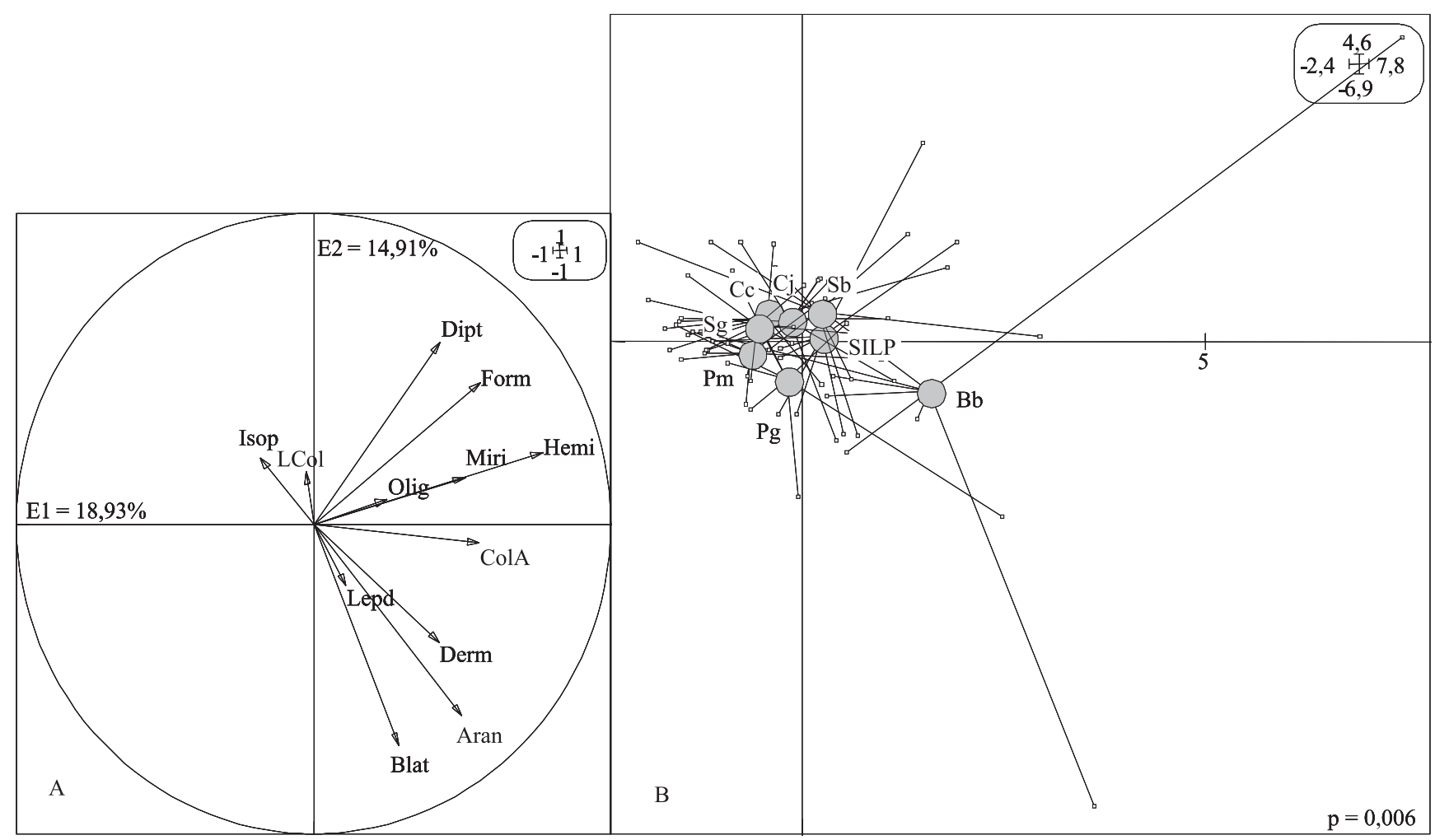

Figura 1. Círculo de correlações entre os grupos da macrofauna (A) e diagrama de ordenação das amostras, em função das plantas de cobertura (B). Form: Formicidae; Olig: Oligochaeta; Lepd: Lepdoptera; ColA: Coleoptera adulto; LCol: larva de Coleoptera; Hemi: Hemiptera; Derm: Dermaptera; Blat: Blattodea; Aran: Araneae; Isop: Isoptera; Miri: Miriapoda; Dipt: Diptera (larva); Bb: braquiária (Brachiaria brizantha cv. Marandu); Cc: guandu-anão (Cajanus cajan (L.) Millisp.); Pg: milheto (Pennisetum glaucum (L). R. Br. cv. BN-2); Pm: capim-mombaça (Panicum maximum cv. Mombaça); Sb: sorgo granífero (Sorghum bicolor (L.) Moench cv. BR 304); Sg: estilosantes (Stylosanthes guianensis cv. Mineirão). 
Isoptera e larva de Coleoptera estão associados aos tratamentos crotalária e guandu.

No diagrama de ordenação (Figura 2 A), tem-se o agrupamento das espécies em função da família onde houve separação significativa entre as leguminosas e as gramíneas $(\mathrm{p}=0,024)$. A maior densidade $(58 \%)$ foi observada nas áreas sob cultivo com leguminosas, das quais se destaca a crotalária, que apresentou $16,3 \%$ da densidade total. A maior densidade de macrofauna nas áreas sob plantio com leguminosas indica preferência alimentar destes organismos pelas plantas de cobertura pertencentes a esta família, o que pode estar relacionado à sua baixa relação $\mathrm{C} / \mathrm{N}$ (Silva et al., 2007). Dias et al. (2007) observaram que a introdução de leguminosas perenes (Mimosa artemisiana e Mimosa tenuiflora), em pastagem de Brachiaria brizantha, favoreceu a presença de Oligochaeta, o que corrobora os resultados do presente estudo.

Os tratamentos com plantas leguminosas apresentaram, em média, 251 indivíduos $\mathrm{m}^{-2}$ do grupo Formicidae, enquanto os tratamentos pertencentes às gramíneas apresentaram em média 143 indivíduos $\mathrm{m}^{-2}$, o que evidencia a preferência deste grupo taxonômico pelo forrageamento das plantas leguminosas. Merlim et al. (2005), ao estudar a macrofauna edáfica em áreas sob cultivo de figo, sobre diferentes coberturas do solo (cobertura viva de grama-batatais, cobertura viva de sirato e cobertura morta da palhada de grama-batatais), observaram que mais de $80 \%$ da fauna edáfica, identificada em grama-batatais, eram pertencentes ao grupo Formicidae, o que caracterizou a mais baixa diversidade funcional entre as coberturas do solo avaliadas.

No diagrama de ordenação (Figura 2 B), tem-se a comparação entre as épocas de amostragem. Observase que há uma separação significativa entre as duas épocas ( $\mathrm{p}<0,001)$ e que a amostragem de setembro, sob cultivo de feijão irrigado, apresentou maior abundância da macrofauna.

A maior variação da densidade, em função da época, foi observada nos grupos Formicidae, Oligochaeta, Coleoptera adulto, Hemiptera e Dermaptera (Tabela 1). A maior densidade foi observada em setembro, porém o número de grupos taxonômicos presentes foi o mesmo para as duas épocas. Em diversos trabalhos (Lavelle et al., 2006; Baretta et al., 2007) foi relatado que, entre outros fatores, a temperatura é determinante para a macrofauna do solo e influencia em sua dinâmica populacional. De acordo com Lavelle (1988), em regiões

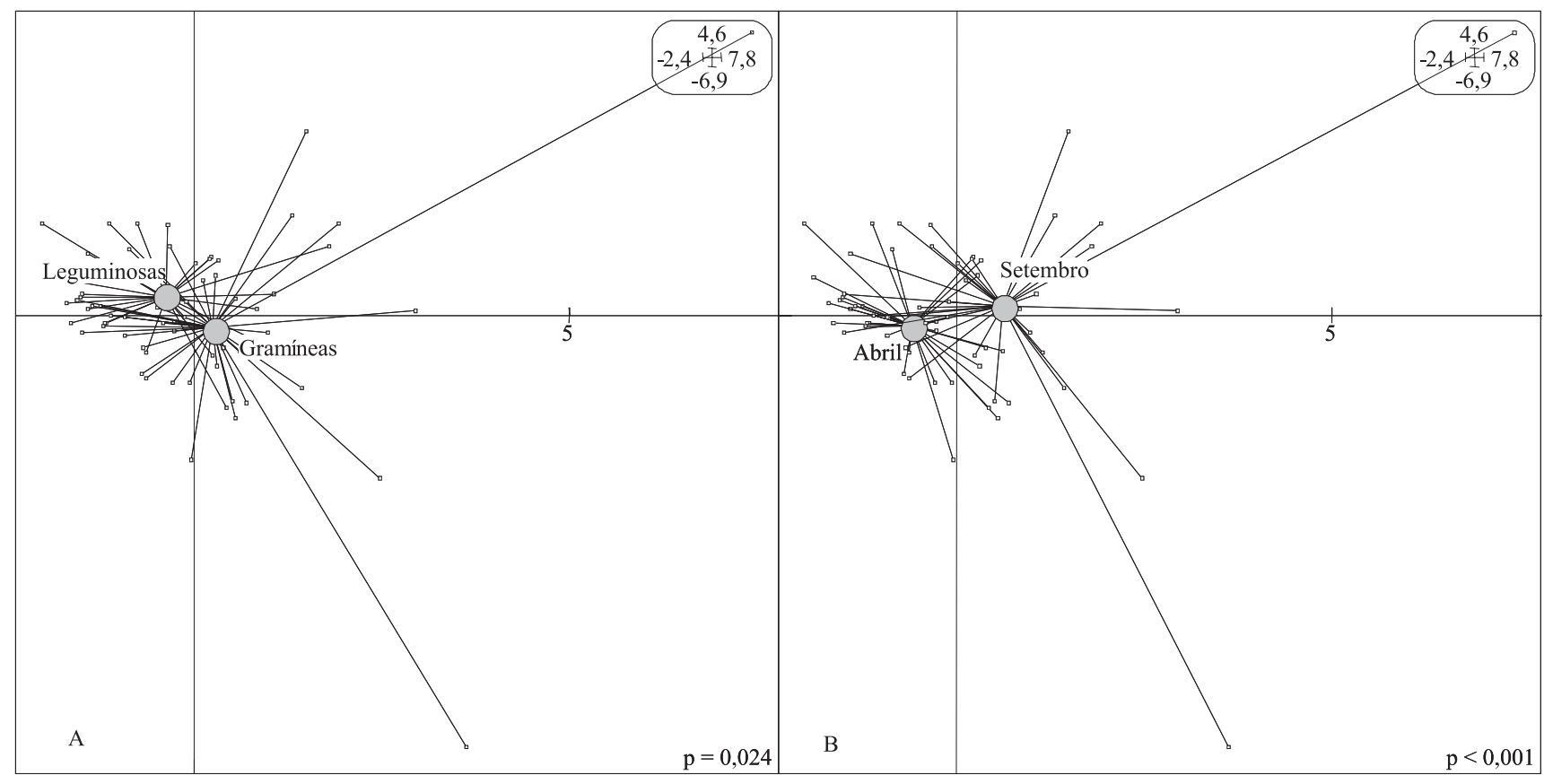

Figura 2. Diagramas de ordenação das amostras, em função da família das plantas de cobertura (A) e da época de amostragem (B). 
tropicais, a macrofauna do solo deve ser amostrada durante e até o final da estação chuvosa, quando os fatores climáticos temperatura e umidade ainda não são limitantes. Melhores condições de umidade do solo, temperatura e estágio de decomposição da cobertura morta, na avaliação de setembro, podem ter favorecido a macrofauna, principalmente dos grupos Formicidae e Oligochaeta. Em setembro, esses dois grupos somaram 3.660 indivíduos $\mathrm{m}^{-2}$, o que representou $63,4 \%$ da macrofauna total identificada nesse período de amostragem. Na mesma avaliação, o grupo Formicidae foi o que apresentou a maior densidade, com 2.332 indivíduos $\mathrm{m}^{-2}$ (40,4\%), seguido pelo grupo Oligochaeta com 23\%. Em razão da baixa densidade, o grupo Isoptera não foi influenciado pela época. Apesar de sua grande abundância em solos do Cerrado (Benito et al., 2004; Silva et al., 2007), observou-se, neste trabalho, baixa densidade do grupo Isoptera (cupins) nas duas épocas de coleta.

\section{Conclusões}

1. Os grupos taxonômicos mais abundantes, em ordem decrescente de densidade relativa, são: Formicidae, Oligochaeta, Dermaptera, Coleoptera adulto, larva de Coleoptera, Hemiptera, Miriapoda, Isoptera, Araneae, Lepidoptera, Blattodea e larvas de Diptera.

2. Há efeito significativo das plantas de cobertura sobre os grupos taxonômicos e densidade relativa da macrofauna edáfica.

3. As plantas de cobertura da família leguminosa favorecem maior densidade relativa de invertebrados do solo, e as gramíneas favorecem os grupos da serrapilheira.

4. A densidade relativa dentro de cada grupo taxonômico varia com a época de coleta.

5. A leguminosa crotalária apresenta maior densidade da macrofauna, seguida pela braquiária solteira, braquiária em consórcio com milho, sorgo, estilosantes, guandu, milheto e mombaça.

\section{Agradecimentos}

Ao Conselho Nacional de Desenvolvimento Científico e Tecnológico, à Embrapa Arroz e Feijão, à Universidade Federal de Goiás e ao Institut de Recherche pour le Développement (IRD), pelo apoio logístico e financeiro.

\section{Referências}

ALCÂNTARA, F.A.; FURTINI NETO, A.E.; PAULA, M.B.; MESQUITA, H.A.; MUNIZ, J.A. Adubação verde na recuperação da fertilidade de um Latossolo Vermelho-Escuro degradado. Pesquisa Agropecuária Brasileira, v.35, p.277-288, 2000.

ALVARENGA, R.C.; COSTA, L.M.; MOURA FILHO, W.; REGAZZI, A.J. Características de alguns adubos verdes de interesse para a conservação e recuperação de solos. Pesquisa Agropecuária Brasileira, v.30, p.175-185, 1995.

AMÁBILE, R.F.; FANCELli, A.L.; CARVAlHO, A.M. Comportamento de espécies de adubos verdes em diferentes épocas de semeadura e espaçamentos na região dos Cerrados. Pesquisa Agropecuária Brasileira, v.35, p.47-54, 2000.

ANDERSON, J.M.; INGRAM, J.S.I. Tropical soil biology and fertility: a handbook of methods. 2.ed. Wallingford: $\mathrm{CAB}$ International, 1993. 221p.

BARETTA, D.; BROWN, G.G.; JAMES, S.W.; CARDOSO, E.J.B.N. Earthworm populations sampled using collection methods in Atlantic forests with Araucaria angustifolia. Scientia Agricola, v.64, p.384-392, 2007.

BARROS, E.; NEVES, A.; BLANCHART, E.; FERNANDES, E.C.M.; WANDELLI, E.; LAVELLE, P. Development of the soil macrofauna community under silvopastoral and agrosilvicultural systems in Amazonia. Pedobiologia, v.47, p.273-280, 2003.

BENITO, N.P.; BROSSARD, M.; PASINI, A.; GUIMARÃES, M.F.; BOBILLIER, B. Transformations of soil macroinvertebrate populations after native vegetation conversion to pasture cultivation (Brazilian Cerrado). European Journal of Soil Biology, v.40, p.147154, 2004.

BLANCHART, E.; VILLENAVE, C.; VIALLATOUX, A.; BARTHÈS, B.; GIRARDIN, C.; AZONTONDE, A.; FELLER, C. Long-term effect of a legume cover crop (Mucuna pruriens var. utilis) on the communities of soil macrofauna and nematofauna, under maize cultivation, in Southern Benin. European Journal of Soil Biology, v.42, 136-144, 2006.

BOER, C.A.; ASSIS, R.A.; SILVA, G.P.; BRAZ, A.J.B.P.; BARROSO, A.L.L.; CARGNELUTTI FILHO, A.; PIRES, F.R. Ciclagem de nutrientes por plantas de cobertura na entressafra em um solo de cerrado. Pesquisa Agropecuária Brasileira, v.42, p.1269-1276, 2007.

CONSTANTINO, R. Padrões de diversidade e endemismo de térmitas no bioma Cerrado. In: SCARIOT, A.; SILVA, J.C.S.; FELFILI, J.M. (Ed.). Cerrado: ecologia, biodiversidade e conservação. Brasília: Ministério do Meio Ambiente, 2005. p.319-333.

DIAS, P.F.; SOUTO, S.M.; CORRÊIA, M.E.F.; ROCHA, G.P.; MOREIRA, J.F.; RODRIGUES, K.D.M.; FRANCO, A.A. Árvores fixadoras de nitrogênio e macrofauna do solo em pastagem de híbrido de Digitaria. Pesquisa Agropecuária Brasileira, v.41, p.1015-1021, 2006.

DIAS, P.F.; SOUTO, S.M.; CORRÊIA, M.E.F.; RODRIGUES, K.M.; FRANCO, A.A. Efeito de leguminosas arbóreas sobre a macrofauna do solo em pastagem de Brachiaria brizantha cv. Marandu. Pesquisa Agropecuária Tropical, v.37, p.38-44, 2007. 
EMBRAPA. Sistema brasileiro de classificação de solos. 2.ed. Rio de Janeiro: Embrapa Solos, 2006. 306p.

DIAS, V.S.; BROSSARD, M.; LOPES-ASSAD, M.L. Macrofauna edáfica invertebrada em áreas de vegetação nativa da região de cerrados. In: LEITE, L.L.; SAITO, C.H. (Ed.). Contribuição ao conhecimento ecológico do Cerrado. Trabalhos selecionados do 3 Congresso de Ecologia do Brasil, Universidade de Brasília, Departamento de Ecologia, 1997. p.168-173.

LAVELLE, P. Assessing the abundance and role of invertebrate communities in tropical soils: aims and methods. Journal of African Zoology, v.102, p.275-283, 1988.

LAVELLE, P.; DECAËNS, T.; AUBERT, M.; BAROT, S.; BLOUIN, M.; BUREAU, F.; MARGERIE, P.; MORA, P.; ROSSI, J.P. Soil invertebrates and ecosystem services. European Journal of Soil Biology, v.42, p.3-15, 2006.

LAVELLE, P.; PASHANASI, B.; CHARPENTIER, F.; GILOT, C.; ROSSI, J.P.; DEROUARD, L.; ANDRÉ, J.; PONGE, J.F.; BERNIER, N. Large-scale effects of earthworms on soil organic matter and nutrient dynamics. In: EDWARDS, C.A. (Ed.). Earthworm Ecology. Boca Raton: St Lucie Press, 1997. p.103-122.

LAVELLE, P.; SPAIN, A.V. Soil ecology. Amsterdam: Kluwer Scientific Publications, 2001. 654p.
LEE, K.E. The biodiversity of soil organisms. Applied Soil Ecology, v.1, p.251-254, 1994.

MARCHÃO, R.L. Integração lavoura-pecuária num Latossolo do Cerrado: impacto na física, matéria orgânica e macrofauna. 2007. 153p. Tese (Doutorado) - Universidade Federal de Goiás, Goiânia.

MENEZES, L.A.S.; LEANDRO, W.M. Avaliação de espécies de coberturas do solo com potencial de uso em sistema de plantio direto. Pesquisa Agropecuária Tropical, v.34, p.173-180, 2004.

MERLIM, A.O.; GUERRA, J.G.M.; JUNQUEIRA, R.M.; AQUINO, A.M. Soil macrofauna in cover crops of figs grown under organic management. Scientia Agricola, v.62, p.57-61, 2005.

SILVA, R.F.; AQUINO, A.M.; MERCANTE, F.M.; GUIMARÃES, M.F. Macrofauna invertebrada do solo sob diferentes sistemas de produção em Latossolo da região do Cerrado. Pesquisa Agropecuária Brasileira, v.41, p.697-704, 2006.

SILVA, R.F.; TOMAZI, M.; PEZARICO, C.R.; AQUINO, A.M.; MERCANTE, F.M. Macrofauna invertebrada edáfica em cultivo de mandioca sob sistemas de cobertura do solo. Pesquisa Agropecuária Brasileira, v.42, p.865-871, 2007.

THIOULOUSE, J.; CHESSEL, D.; DOL ${ }^{2}$ DEC, S.; OLIVIER J.M. ADE-4: a multivariate analysis and graphical display software. Statistics Computing, v.7, p.75-83, 1997. 